\title{
Roles of ADF/cofilin in actin polymerization and beyond
}

\section{James R Bamburg* and Barbara W Bernstein}

\author{
Address: Department of Biochemistry and Molecular Biology, 1870 Campus Delivery, Colorado State University, Fort Collins, \\ CO 80523-1870, USA \\ *Corresponding author: James R Bamburg (jbamburg@lamar.colostate.edu) \\ FI000 Biology Reports 2010, 2:62 (doi:10.34I0/B2-62)
}

The electronic version of this article is the complete one and can be found at: http://fl000.com/reports/biology/content/2/62

\begin{abstract}
In collaboration or competition with many other actin-binding proteins, the actin-depolymerizing factor/cofilins integrate transmembrane signals to coordinate the spatial and temporal organization of actin filament assembly/disassembly (dynamics). In addition, newly discovered effects of these proteins in lipid metabolism, gene regulation, and apoptosis suggest that their roles go well beyond regulating the cytoskeleton.
\end{abstract}

\section{Introduction and context}

All eukaryotes express at least one member of the essential actin-depolymerizing factor (ADF)/cofilin family of actin-binding proteins [1]. Three forms are expressed in mammals: ADF (also known as destrin); cofilin-1, the major ubiquitous form in non-muscle tissue; and cofilin-2, the major form in differentiated muscle. In vitro, ADF and cofilin-1 are qualitatively similar in many of their actin-dynamizing activities and regulation but differ quantitatively. ADF has a weaker nucleating ability than cofilin and thus can serve as a monomer-sequestering protein for ATP-actin at concentrations in which cofilin would enhance assembly of the cofilin-ATP-actin complex $[2,3]$. However, at the physiological molar ratios to actin (generally $1: 25$ to $1: 4$ ), both ADF and cofilin bind ADP subunits in filamentous actin (F-actin) and sever filaments, leading to enhanced actin dynamics [2]. Silencing and rescue experiments of ADF and cofilin in cultured cells show that either protein can rescue defects in cytokinesis and cell motility [4], although there are suggestions in the literature that the two proteins differ in their ability to regulate cell migration in three-dimensional matrices, with ADF being the more important protein for cell invasion through Matrigel [5]. Knockout of the cofilin-1 gene in mice is embryonic lethal [6], whereas the only gross deficit in ADF knockout mice is postnatal blindness due to corneal thickening at about 4 weeks [7]. Because cofilin-1, the most ubiquitous of the isoforms, is usually expressed at higher levels and has been studied more intensely because of its essential role in development, this review will focus on cofilin-1, hereafter just called cofilin.

\section{Major recent advances \\ Mechanism and function in actin dynamics}

Cofilin is best known as a regulator of actin filament non-equilibrium assembly/disassembly. Whether cofilin promotes actin assembly or disassembly depends upon the concentration of cofilin relative to actin and the relative concentrations of other actin-binding proteins $[1,8]$. In vitro studies have demonstrated that if the ratio of cofilin/actin subunits in a filament is low (less than 1\%), this results in persistent filament severing (Figure 1) [8]. At higher cofilin/actin molar ratios $(1: 10$ to $1: 2)$, cofilin severs rapidly but transiently because it binds F-actin cooperatively and stabilizes F-actin in a twisted form as it saturates the severed pieces. Indeed, the assembly of the cofilinADP-actin complex into non-dynamic actin bundles (also called rods) is an important energy-conserving mechanism that in most cells is readily reversible (Figure 1); however, in axons and dendrites of stressed neurons, the rods block transport and cause loss of synapses [9], perhaps contributing to dementias, including Alzheimer disease [10]. 
Figure I. Concentration-dependent effects of cofilin on actin dynamics
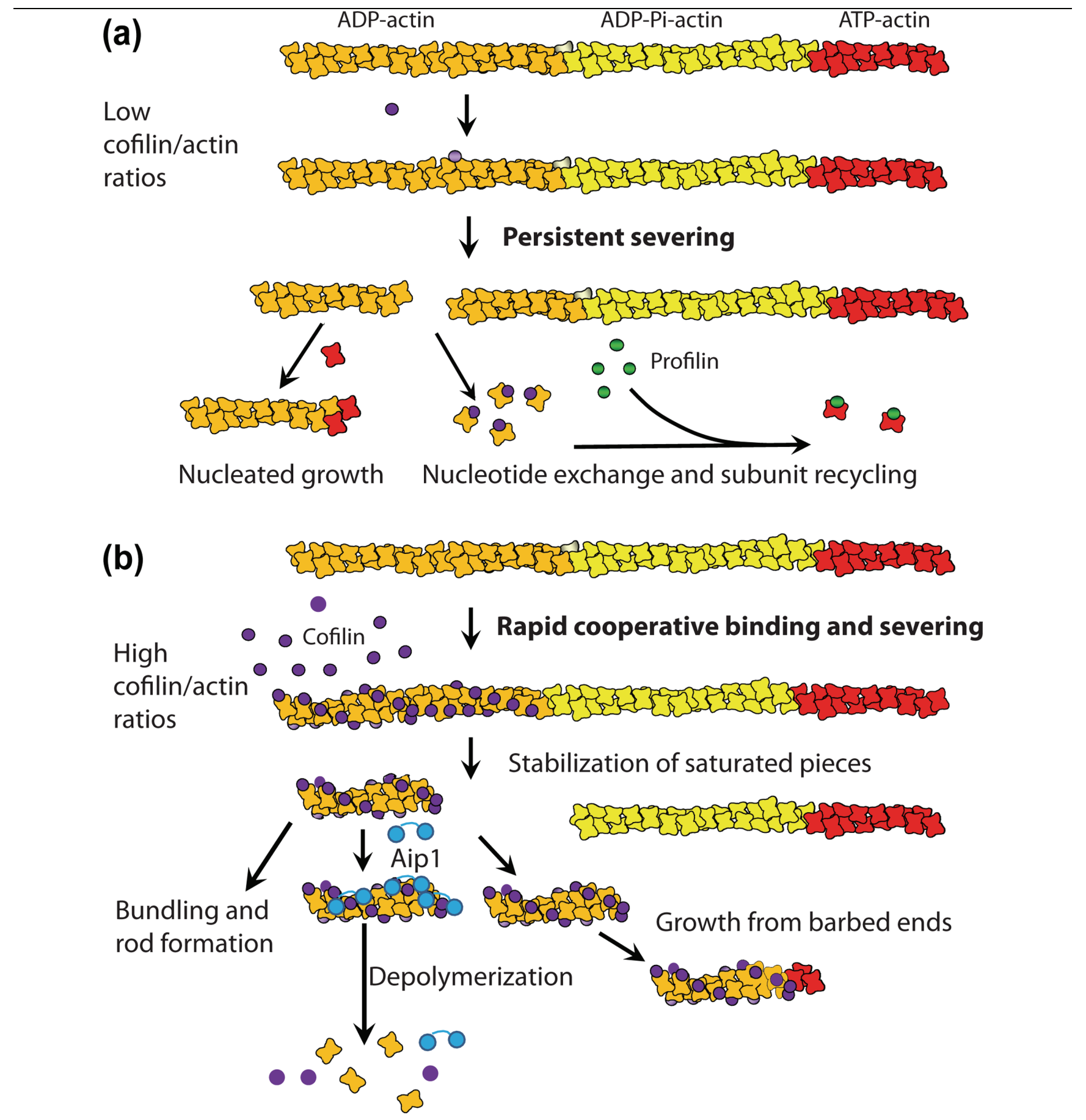

(a) Cofilin (purple) binds preferentially to ADP-actin (orange) and, at low stoichiometry with respect to actin subunits, severs filaments, creating new barbed and pointed ends. The cofilin dissociates with an actin subunit in the ADP form, and nucleotide exchange, enhanced by Srv2/CAPI (exchange factor for actinbound nucleotide when complexed to cofilin) and/or profilin (green), occurs on the actin. Cofilin can recycle to sever again. The pieces of filamentous actin (F-actin) generated can nucleate filament growth or can enhance depolymerization if assembly-competent ATP-actin is limiting. (b) At higher stoichiometry, cofilin binds to ADP-actin, but since binding is cooperative, regions of the F-actin become saturated and stabilized in the 'twisted form'. Severing occurs rapidly, but as the cofilin is sequestered on the pieces of actin, severing is not persistent. Fragments are further depolymerized in the presence of actininteracting protein I (Aip I) (blue) to generate monomer or can be used to nucleate growth. In cells under stress where ADP-actin levels are elevated, the cofilin-saturated F-actin assembles into rod-shaped bundles. 
Other actin-binding proteins may enhance, modulate, or eliminate cofilin effects on actin dynamics. Among the best studied of these are actin-interacting protein 1 (Aip1) (also known as WDR1), tropomyosins, cortactin, actin-related protein 2 and 3 (Arp2/3) complex, coronins, and Srv2/CAP1 (exchange factor for actin-bound nucleotide when complexed to cofilin). Aip1 binds to cofilin-actin filaments and enhances the severing and depolymerizing activity of cofilin, potentiating the generation of actin monomers [11]. Some of the more than 40 mammalian tropomyosin isoforms have an F-actin-stabilizing function, in which they compete with cofilin for F-actin binding. Surprisingly, however, certain tropomyosin isoforms may enhance cofilin recruitment and F-actin turnover [12].

Cortactin has its highest binding affinity to F-actin subunits containing ATP or ADP- $\mathrm{P}_{\mathrm{i}}$, thus protecting newly added subunits, whereas cofilin has its highest affinity for ADP-actin subunits, which accumulate in the more aged region of the filament. Because cofilin binding to F-actin enhances $P_{i}$ (inorganic phosphate) release from neighboring subunits [13] and cortactin binding resists this, the balanced effect of cofilin and cortactin helps maintain the filament network and its dynamics at the leading edge of a migrating cell [14]. Cortactin is also an activator of the Arp2/3 complex, a seven-protein complex that binds along pre-existing filaments to nucleate new filament growth or capture filament pointed ends, creating $70^{\circ}$ branch points. These branches provide the numerous filament barbed ends, the sites for actin assembly that drive membrane protrusion during polarized cell migration. In addition to cortactin, several proteins activated by transmembrane signaling, including neural Wiskott-Aldrich syndrome protein (N-WASp), have the ability to activate the Arp $2 / 3$ complex. In the highly specialized invadopodium compartment of metastatic cells, cofilin binds cortactin in a complex with Arp2/ 3 , along with the Arp2/3 activator N-WASp and the NWASp activator Nck1 (adaptor molecule with src homology domains 2 and 3) [15]. Cofilin is released for the essential function of severing after phosphorylation of a cortactin tyrosine [16]. Furthermore, as demonstrated only in vitro, cofilin can debranch the Arp2/3 complex by direct interaction [17], again suggesting that cofilin activity must be tightly regulated both spatially and temporally. In fact, the relationship between cofilin level and cell migration is biphasic [18]. A moderate increase in cofilin accelerates cell migration, clearly a factor in metastasis, but greater increases reverse this effect. Cofilin and its upstream regulators are essential to the development of cell polarity and to the maintenance of polarized cell motility [19-21].
Another leading-edge protein is coronin 1 (Crn1), which has three domains: an $\mathrm{N}$-terminal $\beta$ propeller domain that binds F-actin, a middle domain, and a C-terminal coiledcoil (CC) domain that modulates the Arp2/3 complex [22]. The $\beta$ propeller and CC domains are involved in opposing effects on cofilin/actin interaction. Which effect prevails depends upon the nucleotide bound to actin [23]. The CC domain competes with cofilin in binding to ATP/ ADP- $\mathrm{P}_{\mathrm{i}}$-actin, thus reducing the already weak binding of cofilin to this region of F-actin. In filament regions rich in ADP-actin, the $\beta$ propeller domain synergizes cofilin severing. When the dominant inhibitory effects of the CC domain are negated by phosphorylation, $\mathrm{Crn} 1$ can switch from its filament-protective role to its cofilin-synergizing role via the $\beta$ propeller domain.

Although in vitro studies of actin with one or two proteins have given us useful mechanistic information, a more complete picture of how the various proteins function in vivo is obtained when increasingly complex mixtures of purified proteins are used. In one such reconstituted system containing cofilin, Aip1, coronin 1a, and fluorescent actin, a new mechanism for cofilin depolymerization of single actin filaments was revealed [24]. F-actin disassembles in successive bursts not previously described and not observed with cofilin alone. Because cofilin has been shown to disrupt subunits between adjacent helical strands of actin in filaments [25] and Aip1 enhances cofilin severing and depolymerizing activity by binding along cofilin-decorated actin, one plausible mechanism suggested for the bursting activity is weakening of inter-filament strand interactions and the severing and removal of single-stranded actin subunits in a depolymerizing burst [24]. This 'cooperative strand separation' is supported by the fact that cofilin severing by itself does not alter the binding of the F-actin barbed-end capping protein (CapZ) but cofilin severing in the presence of coronin 1a and Aip 1 abrogates CapZ binding. Unwinding of actin filament strands has been observed previously in electron micrographs, though not under the same conditions as used above [26]. However, interference with CapZ binding could arise from other mechanisms, so further studies are required to confirm the strand separation model.

\section{New cellular functions of cofilin unrelated to actin-assembly regulation}

Chaperoning actin to the nucleus

Actin itself has no nuclear localization sequence but cofilin does. Because actin has important functions in chromatin remodeling, formation of heterogeneous nuclear ribonucleoprotein complexes, and gene expression [27], the ability of cofilin to enable actin nuclear 
functions is one of its crucial cellular roles. In higher plants, in which many different isoforms of ADF are expressed, ADF9 deficiencies produced gene expression and chromatin remodeling phenotypes in addition to morphological and cytoskeletal defects reflecting ADF9 cytoplasmic functions [28]. It is not known whether these nuclear functions require actin or are mediated by ADF9 alone.

Release of mitochondrial cytochrome $c$ In lymphoma cells, cofilin oxidation and its mitochondrial translocation were found to induce apoptosis [29]. Cofilin translocation opens the mitochondrial permeability transition pore and releases cytochrome $c$, an early step in apoptosis. Neither the translocation to mitochondria nor the release of cytochrome $c$ requires actin binding.

Activation of phospholipase DI

Cofilin phosphorylated on serine 3 is inactive in binding actin and has always been considered an inactive form.
However, it was recently reported that phospho-cofilin can directly activate phospholipase D1 (PLD1) [30], an enzyme essential for chemotaxis of phagocytic cells (Figure 2). Investigation into the key role of cofilin in cell polarization (development of a leading edge) has previously focused on the release of active cofilin from its inhibition through binding phosphatidylinositol-4,5bisphosphate (PtdIns4,5 $\mathrm{P}_{2}$ ) [1]. This lipid binding occurs in a multivalent manner and acts not only as a PtdIns4,5 $\mathrm{P}_{2}$-density sensor on the membrane [31] but also as a source of potentially active cofilin to generate actin barbed ends in response to signals that cause PtdIns $4,5 \mathrm{P}_{2}$ hydrolysis [14]. The activation of PLD1 by phospho-cofilin increases membrane phosphatidic acid, which is required for the activation of DOCK (dedicator of cytokinesis) proteins, a family of membrane-associated guanine nucleotide exchange factors for Rac1 [32]. Rac1 is also required for polarized cell migration. Because cofilin phosphorylation downstream of Rac1 (via PAK [p21-activated kinase] and LIMK [LIM kinase]) generates phospho-cofilin, this feed-forward

Figure 2. Possible roles of cofilin and phospho-cofilin in the establishment of the leading edge

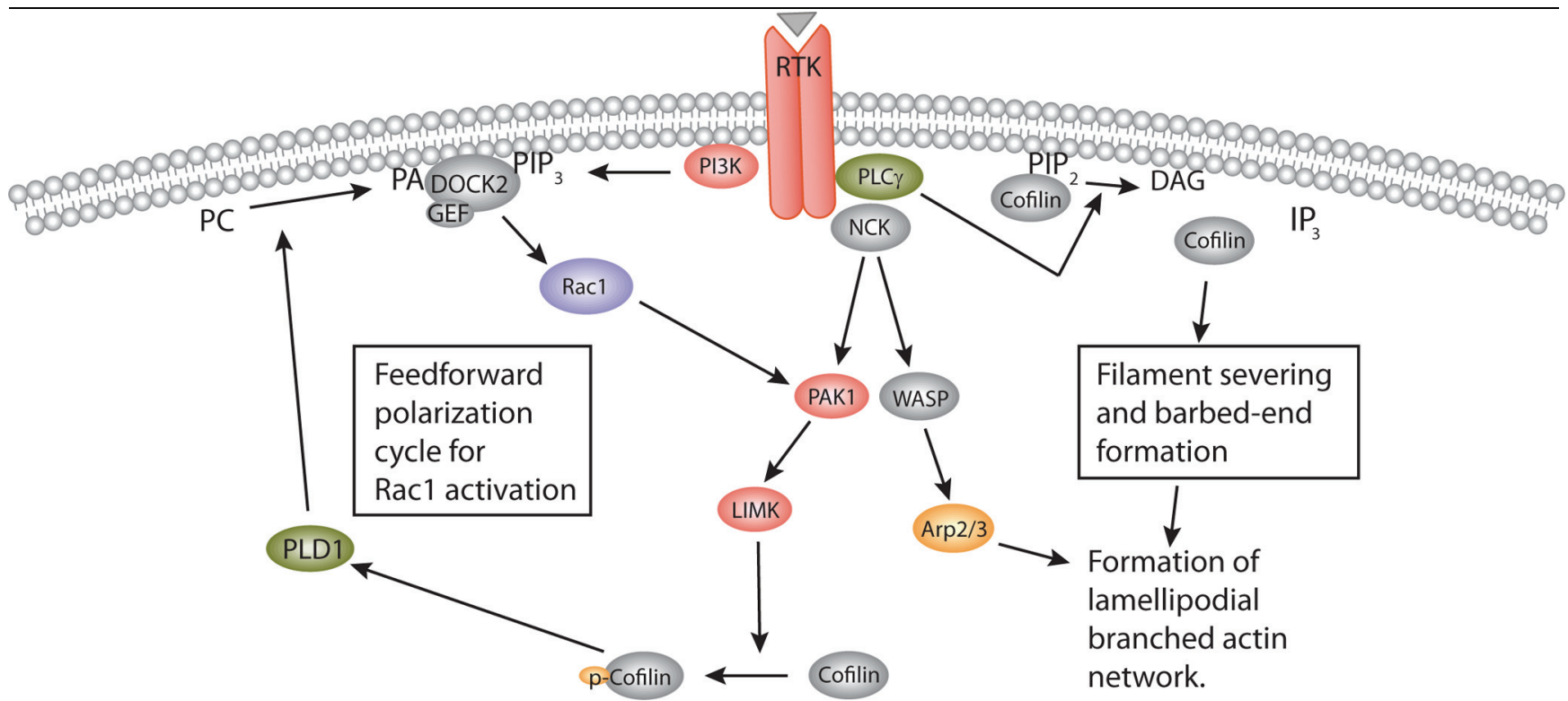

In response to signaling through a receptor tyrosine kinase (RTK), phospholipase C gamma (PLC $\gamma$ ) is activated and hydrolyzes phosphatidylinositol-4,5bisphosphate (Ptdlns4,5 $\mathrm{P}_{2}$ ), releasing active cofilin from its inhibitory binding, allowing severing of capped quiescent filaments, and generating free barbed ends for driving assembly. Actin-related protein 2 and 3 (Arp2/3) complex is also activated via Wiskott-Aldrich syndrome protein (WASP) to set up the branched filament network driving forward protrusion of the membrane. The RTK also recruits phosphatidylinositol 3-kinase (PIK), generating Ptdlns3,4,5P 3 , which serves as a docking site for the binding of dedicator of cytokinesis 2 (DOCK2). The Racl guanine nucleotide exchange activity of DOCK2 is exposed only upon binding of the tail of DOCK2 to phosphatidic acid (PA). PA is generated from the hydrolysis of other phospholipids, such as phosphatidylcholine (PC), by the enzyme phospholipase DI (PLDI), which is activated by P-cofilin. Active Racl activates the P2I-activated kinase (PAKI), which activates the cofilin phosphorylation through LIM kinase (LIMK). This feed-forward cycle maintains active Racl at the leading edge but becomes self-limiting when cofilin phosphatases also are recruited or become active through downstream signals from these (e.g., inositol triphosphate $\left[\mathrm{IP}_{3}\right] \rightarrow$ calcium $\rightarrow$ calmodulin $\rightarrow$ calcineurin $\rightarrow$ slingshot phosphatase) and/or other pathways. DAG, diacylglycerol; GEF, guanine nucleotide exchange factor; NCK, adaptor molecule with src homology domains 2 and 3; $\mathrm{PIP}_{2}$, phosphatidyinositol diphosphate; $\mathrm{PIP}_{3}$, phosphatidyinositol triphosphate. 
cycle makes cofilin both an upstream activator and a downstream effector of Rac1 (Figure 2).

\section{New modes of regulation}

Phospho-tyrosine-dependent cofilin turnover

The phosphoregulation of ADF and cofilin on serine 3 is modulated by many transmembrane signaling pathways and their multiple regulatory kinases and phosphatases that converge on this site [1]. However, a recently identified src kinase phosphorylation site on cofilin (Y68), but not ADF (F68), provides another mode of cofilin regulation [33]. Phosphorylation of Y68 does not affect the actin-dynamizing activity of cofilin but does increase its ubiquitination and proteosome degradation to reduce total cofilin levels and cell spreading.

\section{Oxidation}

Cofilin is also regulated by oxidation of its four cysteine residues. In $\mathrm{T}$ cells, the generation of a C39-C80 intramolecular bond, delivered by the oxidative burst of granulocytes, does not eliminate the actin binding of cofilin but does increase F-actin [34]. Further studies were performed on the activity of cofilin oxidized with taurine chloramine, the primary oxidant generated by activated neutrophils [29]. Two intramolecular disulfide bonds form in cofilin in taurine-chloramine-treated lymphoma cells in which cofilin is a main target of oxidation. Neither disulfide bond alone inhibits cofilinactin binding in vitro, but actin binding is eliminated when both internal disulfide bonds form. Furthermore, mutation of any one of these cysteines or serine 3 phosphorylation blocks cofilin's induction of cytochrome $c$ release from mitochondria and inhibits the taurine-chloramine-induced apoptosis.

\section{Future directions}

It remains to be determined whether and exactly how the concentration dependence of active cofilin in actin turnover, stability, and nucleation contributes to the impressive spatial coordination of actin dynamics that underlies highly motile regions such as lamellipodia and neuronal growth cones. Many of the effects of cofilin previously ascribed to its direct modulation of actin may have to be reconsidered if PLD1 is also shown to have a role in mediating the process in question. Indeed, with cofilin being able to function in some cells as both an upstream activator and a downstream effector of Rac1 [10], the interpretation of results from many experiments inter-relating Rac1 and cofilin may need to be reexamined in light of this paradigm.

The transcriptional activity of plant ADF suggests that $\mathrm{ADF} /$ cofilins in animal cells may also regulate gene expression. Both mammalian $\mathrm{ADF}$ and cofilin have proven nuclear localization sequences and have been observed to accumulate in cell nuclei under some conditions. If their nuclear functions turn out to be independent of their ability to transport actin, target genes will need to be identified and many of the cytoplasmic effects of ADF/cofilin activation might need to be re-examined to determine whether they are independent of transcriptional events.

The src kinase phosphorylation of cofilin, but not ADF, enhances its ubiquitination and degradation and suggests a significant potential regulatory difference between cofilin and ADF in cellular processes involving src kinases. Indeed, some of the tumor cell line differences that have been observed in migration assays could result from different levels of ADF and cofilin [5]. Certainly, this regulatory difference suggests that a more detailed analysis of the specific levels of $\mathrm{ADF} / \mathrm{cofilin}$ species is required when studying the roles of these proteins in cellular processes.

Cofilin plays an important role in the development and function of both neuromuscular junctions [35] and dendritic spines, the latter being the major excitatory synapses in the brain [36]. Cofilin regulation is critical for insertion of the AMPA family of glutamate receptors into the spine membrane, which give spines their functional plasticity important in memory and learning [37]. Understanding mechanistically how cofilin modulates cognition requires further study.

Finally, the newly discovered effect of phospho-cofilin on PLD1 activity suggests that additional work is needed to define the role of this enzyme and its cofilin dependence in different cellular processes. PLD1 is reported to be important in the alteration of membrane curvature during vesicle budding, endocytosis, and phagocytosis. Indeed, cofilin inhibition or knockdown has profound effects on the function and dynamics of the Golgi [38]; some may be due to actin regulation and others may be due to PLD1 regulation. It is of interest to determine whether cofilin phosphorylation through transmembrane signaling is important for the regulation of PLD1 in its many reported functions in cell migration and phagocytosis.

\section{Abbreviations}

ADP, adenosine diphosphate; ATP, adenosine triphosphate; ADF, actin-depolymerizing factor; Aip1, actininteracting protein 1 ; Arp2/3, actin-related protein 2 and 3; CapZ, filamentous actin barbed-end capping protein; CC, coiled-coil; Crn1, coronin 1; F-actin, filamentous actin; N-WASp, neural Wiskott-Aldrich syndrome protein; $\mathrm{P}_{\mathrm{i}}$, inorganic phosphate; PLD1, phospholipase D1; PtdIns4,5 $\mathrm{P}_{2}$, phosphatidylinositol-4,5-bisphosphate. 


\section{Competing interests}

The authors declare that they have no competing interests.

\section{Acknowledgments}

This work was supported by grant NS40371 from the National Institute of Neurological Disorders and Stroke of the National Institutes of Health.

\section{References}

I. Van Troys M, Huyck L, Leyman S, Dhaese S, Vandekerkhove J, Ampe C: Ins and outs of ADF/cofilin activity and regulation. Eur J Cell Biol 2008, 87:649-67.

2. Yeoh S, Pope B, Mannherz HG, Weeds AG: Determining the differences in actin binding by human ADF and cofilin. $J \mathrm{Mol}$ Biol 2002, 3 I5:91I-25.

3. Chen H, Bernstein BW, Sneider JM, Boyle JA, Minamide LS, Bamburg JR: In vitro activity differences between proteins of the ADF/cofilin family define two distinct subgroups. Biochemistry 2004, 43:7127-42.

4. Hotulainen P, Paunola E, Vartianen MK, Lappalainen P: Actindepolymerizing factor and cofilin-I play overlapping roles in promoting rapid $\mathbf{F}$-actin depolymerization in mammalian nonmuscle cells. Mol Biol Cell 2005, I 6:649-64.

5. Estornes Y, Gay F, Gevrey J-C, Navoizat S, Nejjari M, Scoazec J-Y, Chayvialle J-A, Saurin J-C, Abellow J: Differential involvement of destrin and cofilin- $I$ in the control of invasive properties of Isreco I human colon cancer cells. Int J Cancer 2007, I 2 I:2 I 62-7I.

FI000 Factor 3.0 Recommended

Evaluated by James Bamburg 06 Sep 2007

6. Gurniak CB, Perlas E, Witke W: The actin depolymerizing factor $\mathbf{n}$-cofilin is essential for neural tube morphogenesis and neural crest cell migration. Dev Biol 2005, 278:23I-4I.

7. Ikeda S, Cunningham LA, Bogess D, Hawes N, Hobson CD, Sundberg JP, Naggert JK, Smith RS, Nishina PM: Aberrant actin cytoskeleton leads to accelerated proliferation of corneal epithelial cells in mice deficient for destrin (actin depolymerizing factor). Hum Mol Genet 2003, I2:1029-37.

8. Andrianantoandro E, Pollard TD: Mechanism of actin filament turnover by severing and nucleation at different concentrations of ADF/cofilin. Mol Cell 2006, 24:13-23.

9. Bamburg JR, Bernstein BW, Davis RC, Flynn KC, Goldsbury C, Jensen JR, Maloney MT, Marsden IT, Minamide LS, Pak CW, Shaw AE, Whiteman I, Wiggan O: ADF/cofilin rods in neurodegenerative diseases. Curr Alzheimer Res 2010, 7:24I-50.

10. Bernstein BW, Bamburg JR: ADF/cofilin: a functional node in cell biology. Trends Cell Biol 2010, 20:187-95.

II. Okreglak V, Drubin DG: Loss of Aipl reveals a role in maintaining the actin monomer pool and an in vivo oligomer assembly pathway. J Cell Biol 2010, 188:769-77.

FI000 Factor 9.0 Exceptional

Evaluated by Pekka Lappalainen 19 Mar 2010

12. Kuhn TB, Bamburg JR: Tropomyosin and ADF/cofilin as collaborators and competitors. Adv Exp Med Biol 2008, 644:232-49.

13. Blanchoin L, Pollard TD: Mechanism of interaction of Acanthamoeba actophorin (ADF/cofilin) with actin filaments. J Biol Chem 1999, 274:I5538-46.

14. Oser M, Condeelis J: The cofilin activity cycle in lamellipodia and invadopodia. J Cell Biochem 2009, 108: I256-62.

15. Desmarais V, Yamaguchi H, Oser M, Soon L, Mouneimne G, Sarmiento C, Eddy R, Condeelis J: N-WASP and cortactin are involved in invadopodium-dependent chemotaxis to EGF in breast tumor cells. Cell Motil Cytoskeleton 2009, 66:303-16.
16. Oser M, Yamaguchi H, Mader CC, Bravo-Cordero Jl, Arias M, Chen X, Desmarais V, Van Rheenen J, Koleske AJ, Condeelis J: Cortactin regulates cofilin and $\mathbf{N}-$ WASp activities to control the stages of invadopodium assembly and maturation. J Cell Biol 2009, 186:57|-87.

FI000 Factor 4.8 Must Read

Evaluated by Matthew Welch 22 Sep 2009, Richard Firtel 29 Sep 2009

17. Chan C, Beltzner CC, Pollard TD: Cofilin dissociates Arp2/3 complex and branches from actin filaments. Curr Biol 2009. 19:537-45.

FI000 Factor 6.0 Must Read

Evaluated by James Bamburg 17 Apr 2009

18. Yap CT, Simpson TI, Pratt T, Price DJ, Maciver SK: The motility of glioblastoma tumour cells is modulated by intracellular cofilin expression in a concentration-dependent manner. Cell Motil Cytoskeleton 2005, 60:153-65.

19. Chen X, Macara IG: Par-3 mediates the inhibition of LIM kinase 2 to regulate cofilin phosphorylation and tight junction assembly. J Cell Biol 2006, I72:67|-8.

FI000 Factor 3.2 Recommended

Evaluated by Pekka Lappalainen 07 Mar 2006, Keith Mostov I4 Mar 2006

20. Wang Y, Du D, Fang L, Yang G, Zhang C, Zheng R, Ullrich A, Lottspeich F, Chen Z: Tyrosine phopshorylated Par3 regulates epithelial tight junction assembly promoted by EGFR signaling. $E M B O J$ 2006, 25:5058-70.

21. Mseka T, Bamburg JR, Cramer LP: ADF/cofilin family proteins control formation of oriented actin-filament bundles in the cell body to trigger fibroblast polarization. J Cell Sci 2007, I 20:4332-44.

22. Cai L, Marshall TW, Uetrecht AC, Schafer DA, Bear JE: Coronin IB coordinates Arp2/3 complex and cofilin activities at the leading edge. Cell 2007, I 28:915-29.

FI000 Factor 6.0 Must Read

Evaluated by James Bamburg 30 Apr 2007

23. Gandhi M, Achard V, Blanchoin L, Goode BL: Coronin switches roles in actin disassembly depending on the nucleotide state of actin. Mol Cell 2009, 34:364-74.

24. Kueh HY, Charras GT, Mitchison TJ, Brieher WM: Actin disassembly by cofilin, coronin, and Aipl occurs in bursts and is inhibited by barbed-end cappers. J Cell Biol 2008, I 82:34I-53.

FI000 Factor 3.0 Recommended

Evaluated by Matthew Welch 12 Aug 2008

25. Bobkov AA, Muhlrad A, Shvetsov A, Benchaar S, Scoville D, Almo SC, Reisler E: Cofilin (ADF) affects lateral contacts in F-actin. J Mol Biol 2004, 337:93-104.

26. McGough A, Chiu W: ADF/cofilin weakens lateral contacts in the actin filament. J Mol Biol 1999, 291:513-19.

27. Zheng B, Han M, Bernier M, Wen JK: Nuclear actin and actinbinding proteins in the regulation of transcription and gene expression. FEBS J 2009, 276:2669-85.

28. Burgos-Rivera B, Ruzicka DR, Deal RB, McKinney EC, King-Reid L, Meagher RB: ACTIN DEPOLYMERIZING FACTOR9 controls development and gene expression in Arabidopsis. Plant Mol Biol 2008, 68:619-32.

29. Klamt F, Zdanov S, Levine RL, Pariser A, Zhang Y, Zhang B, Yu LR, Veenstra TD, Shacter E: Oxidant-induced apoptosis is mediated by oxidation of the actin-regulatory protein cofilin. Nat Cell Biol 2009, I I:| $24 \mid-6$.

FI000 Factor 6.0 Must Read

Evaluated by James Bamburg 13 Oct 2009

30. Han L, Stope MB, de Jesus ML, Oude Weernink PA, Urban M, Wieland T, Rosskopf D, Mizuno K, Jakobs KH, Schmidt M: Direct stimulation of receptor-controlled phospholipase $D I$ by phospho-cofilin. EMBO J 2007, 26:4 I89-202. 
31. Zhao H, Hakala M, Lappalainen P: ADF/cofilin binds phosphoinositides in a multivalent manner to act as a PIP(2)-density sensor. Biophys J 2010, 98:2327-36.

32. Nishikimi A, Fukuhara H, Su W, Hongu T, Takasuga S, Mihara H, Cao O, Sanematsu F, Kanai M, Hasegawa H, Tanaka Y, Shibasaki M, Kanaho Y, Sasaki T, Frohman MA, Fukui Y: Sequential regulation of DOCK2 dynamics by two phospholipids during neutrophil chemotaxis. Science 2009, 324:384-7.

FI000 Factor 4.8 Must Read

Evaluated by Jens V Stein 22 Apr 2009, Steve Ward 24 Apr 2009

33. Yoo $Y, H o H J$, Wang C, Guan JL: Tyrosine phosphorylation of cofilin at Y68 by v-Src leads to its degradation through ubiquitin-proteasome pathway. Oncogene 2009, 29:263-72.

FI000 Factor 3.0 Recommended

Evaluated by James Bamburg 08 Jan 2010

34. Klemke M, Wabnitz GH, Funke F, Funk B, Kirchgessner H, Samstag Y: Oxidation of cofilin mediates $\mathrm{T}$ cell hyporesponsiveness under oxidative stress conditions. Immunity 2008, 29:404-I3.
35. Lee CW, Han J, Bamburg JR, Han L, Lynn R, Zheng JQ: Regulation of acetylcholine receptor clustering by ADF/cofilin-directed vesicular trafficking. Nat Neurosci 2009, 12:848-56.

FI000 Factor 8.2 Exceptional

Evaluated by David Ginty 09 Jul 2009, H Benjamin Peng 22 Jul 2009, Bettina Winckler II Nov 2009

36. Carlisle HJ, Manzerra P, Marcora E, Kennedy MB: SynGAP regulates steady-state and activity-dependent phosphorylation of cofilin. J Neurosci 2008, 28: 13673-83.

37. Yuen EY, Liu W, Kafri T, Van Praag H, Yan Z: Regulation of AMPA receptor channels and synaptic plasticity by cofilin phosphatase slingshot in cortical neurons. J Physiol 2010, 588:266I-7I.

38. von Blume J, Duran JM, Forlanelli E, Alleaume AM, Egorov M, Polishchuk R, Molina H, Malhotra V: Actin remodeling by ADF/ cofilin is required for cargo sorting at the trans-Golgi network. J Cell Biol 2009, 187:1055-69.

FI000 Factor 3.0 Recommended

Evaluated by Pekka Lappalainen 20 Jan 2010 